\title{
Doenças Reumáticas Auto-Imunes em Indivíduos Infectados pelo HTLV-1
}

\section{Autoimmune Rheumatic Diseases in HTLV-1 Infected Individuals}

\author{
Mônica Martinelli Nunes de Carvalho( ${ }^{(1)}$, Albino E. Novaes ${ }^{(2)}$, Edgar Marcelino de Carvalho ${ }^{(3)}$, \\ Maria Ilma Araújo(4)
}

\section{RESUMO}

O HTLV-1 foi o primeiro retrovírus humano a ser associado às doenças malignas leucemia e linfoma de células $\mathrm{T}$ do adulto (LLTA). Ele está relacionado também a uma doença inflamatória crônica do sistema nervoso central (SNC) conhecida como paraparesia espástica tropical/mielopatia associada ao HTLV-1 (PET/MAH). O HTLV-1 tem sido implicado na patogênese de várias doenças auto-imunes, tais como: diabetes, esclerose múltipla, dermatite infectiva, uveíte e artropatia. Ao longo dos anos, a infecção retroviral tem assumido um importante papel na patogênese das doenças reumáticas auto-imunes. Partículas semelhantes aos retrovírus têm sido identificadas em tecidos de pacientes com artrite reumatóide (AR), síndrome de Sjögren, lúpus eritematoso sistêmico (LES) e polimiosite. A síndrome de Sjögren e a AR têm sido as doenças reumáticas mais encontradas nos pacientes infectados pelo HTLV-1, sendo a freqüência mais elevada nos pacientes com mielopatia. A alta prevalência de síndrome de Sjögren e de AR entre os indivíduos com mielopatia sugere que a carga viral e a resposta inflamatória exacerbada, que concorrem para o desenvolvimento da mielopatia, devem também influenciar no desenvolvimento das doenças reumáticas auto-imunes.

Palavras-chave: HTLV-1, doenças reumáticas, doenças autoimunes.

\section{INTRODUÇÃO}

Recentes estudos na virologia molecular e imunologia do HTLV-1 mostram a importância da resposta imune do hospedeiro na evolução clínica de doenças associadas à infecção pelo HTLV-l e buscam explicações para o fato de que alguns portadores do vírus desenvolvem doenças graves, enquanto a maioria permanece saudável ao longo da vida ${ }^{(1)}$. Nos indivíduos que desenvolvem doenças, estas

\begin{abstract}
The HTLV-I was the first human retrovirus associated with adult T-cell leukemia/lymphoma (LLTA). The virus also causes a chronic inflammatory disease of the central nervous system named HTLV-1-associated myelopathy or tropical spastic paraparesis (HAM/TSP). HTLV-1 has been implicated in the pathogenesis of many autoimmune diseases, such as diabetes, multiple sclerosis, infective dermatitis, uveitis and arthropathy. It has long been suggested that retroviral infection may play a role in the pathogenesis of autoimmune rheumatic diseases. Particles resembling retroviruses have been reported in tissue from patients with rheumatoid arthritis (RA), Sjögren's syndrome, systemic lupus erythematosus (SLE) and polymyositis. Sjögren's syndrome and $R A$ have been the most frequent rheumatic diseases in patients infected with HTLV-1, being more prevalent in patients with myelopathy. The high prevalence of Sjögren's syndrome and $R A$ in individuals with myelopathy, suggests that the viral burden and exacerbated inflammatory response may play a role not only in the development of myelopathy, but may also influence the outcome of autoimmune rheumatic diseases.
\end{abstract}

Keywords: HTLV-1, rheumatic diseases, autoimmune diseases.

podem ser incapacitantes e fatais e ainda não se dispõe de vacinas ou tratamento satisfatório para a infecção e nenhuma maneira de prevenir o desenvolvimento de doenças graves nas pessoas infectadas. Carvalho et $a^{(2)}$ demonstraram que células de portadores do HTLV-l sem manifestações neurológicas secretam altos níveis de IFN-G TNF-A, IL5 e IL-10 in vitro na ausência de estímulo, comparados com células de doadores de sangue soronegativos para o

\footnotetext{
Serviço de Imunologia do Hospital Universitário Professor Edgard Santos (HUPES), Universidade Federal da Bahia (UFBA). Recebido em 20/12/05. Aprovado, após revisão, em 14/06/06.

1. Especialista em Reumatologia, Mestre em Imunologia e Professora da UFBA.

2. Chefe do Serviço de Reumatologia do HUPES da UFBA.

3. Professor Livre Docente da UFBA, Chefe do Serviço de Imunologia do HUPES

4. Professora Adjunta da Escola Bahiana de Medicina e Saúde Pública e Pesquisadora do Serviço de Imunologia do HUPES da UFBA.

Endereço para correspondência: Mônica Martinelli Nunes de Carvalho, Serviço de Imunologia do Hospital Universitário Professor Edgard Santos, Rua João das Botas, s/n, Canela, CEP 40110-160, Salvador, BA, Brasil, telefone: (71) 3237-7353, fax: (71) 3245-7110, e-mail : monicamartinelli@atarde.com.br
} 
HTLV-1. Como o vírus induz também no sistema imune uma resposta proliferativa linfocitária exuberante, tem-se aventado a possibilidade de que doenças auto-imunes sejam mais freqüentes nestes indivíduos ${ }^{(3)}$. Existem na literatura dados que sugerem um aumento de manifestações articulares entre indivíduos infectados pelo HTLV-1 ${ }^{(4,5,6)}$. No Brasil, particularmente, na cidade de Salvador (BA), a prevalência da infecção pelo HTLV-1 é de aproximadamente $1,8 \%$, sendo uma das maiores do País ${ }^{(7)}$, e ainda não existem dados na literatura relacionados à freqüência de doenças associadas ao HTLV-1 nesta população.

Neste trabalho serão discutidas as principais doenças reumáticas auto-imunes associadas à infecção pelo HTLV-1 de acordo com o que vem sendo publicado sobre o assunto.

\section{MECANISMOS DE AUTO-IMUNIDADE INDUZIDOS PELO HTLV-1 IMPLICADOS NO DESENVOLVIMENTO DE ARTROPATIAS}

Tem sido descrito que a infecção pelo HTLV-1 induz a expressão de moléculas do complexo principal de histocompatibilidade ( $\mathrm{CPH} / \mathrm{MHC})$ de classe I e II em células neuroblastômicas ${ }^{(8)}$. Aono et a ${ }^{(9)}$ observaram indução similar de moléculas de MHC em células sinoviais infectadas pelo HTLV-1. A alta expressão de MHC e conseqüente aumento na apresentação de antígenos na sinóvia, além da alta produção de citocinas pró-inflamatórias, induzidas pelo HTLV-1, sugere a participação do vírus no desenvolvimento de auto-imunidade e artropatia em indivíduos infectados por este vírus ${ }^{(10)}$. Foi demonstrado, por exemplo, que o gene regulatório tax do HTLV-1 pode ativar muitos genes relacionados à resposta imune, tais como, os genes da IL-1, IL-2, do receptor de IL-1 e da IL-6. O aumento da expressão de IL-6 por células sinoviais tem contribuído para a fisiopatologia da artrite relacionada ao HTLV-1 $\mathbf{l}^{(11)}$. Saijo et a ${ }^{(12)}$ demonstraram que a ativação de célula $\mathrm{T}$ pela IL-1 é importante para o desenvolvimento de autoimunidade e artrite em camundongos transgênicos. Outros estudos em camundongos transgênicos sugerem que os elevados níveis de IL-2 previnem a apoptose das células T auto-reativas, mediada pelo Fas na periferia, e isso representaria um mecanismo importante para o desenvolvimento de artropatia auto-imune ${ }^{(13)}$. Um outro mecanismo envolvido na patogenicidade da artropatia inflamatória semelhante à artrite reumatóide (AR), desenvolvida em camundongos transgênicos carreando a região env-pX do HTLV-1, é a quebra de tolerância para auto-antígenos presentes nas articulações afetadas, gerando expansão oligoclonal de células $\mathrm{T}^{(14)}$. O gene tax do HTLV-1, como demonstrado por Khoa et $a^{\left({ }^{(5)}\right.} \mathrm{em}$ modelo experimental, pode também ativar um outro gene denominado de HOXD9 envolvido no desenvolvimento de artrite.

Dentre as várias explicações para o fenômeno de auto-imunidade em indivíduos infectados pelo HTLV-1, encontra-se também o mimetismo molecular, caracterizado por uma resposta imune ao agente infeccioso que reage cruzadamente com antígenos do hospedeiro, resultando em doença. Esta hipótese tem sido implicada na patogênese do diabetes, do lúpus e da esclerose múltipla em indivíduos infectados pelo vírus ${ }^{(16)}$.

\section{MANIFESTAÇÕES REUMÁTICAS NA INFECÇÃO PELO HTLV-1}

\section{ARTRITE COMO MANIFESTAÇÃO DA LEUCEMIA DE CÉLULAS T DO ADULTO}

A artrite relacionada com a leucemia de células $\mathrm{T}$ do adulto (LTA) caracteriza-se por poliartrite envolvendo articulações interfalangianas proximais (IFP), punhos, joelhos e tornozelos. Pode ser ou não erosiva semelhante à $\mathrm{AR}^{(17)}$ e preceder o quadro de LTA em anos ${ }^{(4)}$.

Harden et $a^{(18)}$ propuseram que um mecanismo patogênico para o desenvolvimento da artrite associada ao LTA seria a migração dos linfócitos leucêmicos ou infectados pelo HTLV-1 para a sinóvia.

\section{ARTROPATIA ASSOCIADA AO HTLV-1}

A artropatia associada ao HTLV-1 (APAH) caracterizase por uma oligoartrite crônica de grandes articulações ${ }^{(19,20)}$ de padrão semelhante à $\mathrm{AR}^{(21)}$. $\mathrm{O}$ fator reumatóide $(\mathrm{FR})$ pode estar positivo na APAH e poderá ocorrer hipergamaglobulinemia. Anticorpos contra antígenos do HTLV-1 podem ser encontrados no soro e no líquido sinovial destes pacientes $^{(22)}$. A histologia sinovial mostra infiltração por linfócitos $\mathrm{T}$ com núcleos típicos daqueles infectados pelo HTLV-1 ${ }^{(19)}$.

Yin et $a^{(23)}$ demonstraram que o HTLV-1 tem tropismo para as células CD68+ de linhagem macrofágica da sinóvia e que essas células, uma vez infectadas pelo HTLV-1, induziriam à produção de TNF-A, aumentando a proliferação sinovial e, consequentemente, levaria ao quadro de artropatia associada ao HTLV-1.

Com relação ao tratamento da APAH, têm sido utilizadas medidas terapêuticas semelhantes àquelas para $\mathrm{AR}$, tais como medicações antiinflamatórias não-hormonais 
(AINHs) para melhora da dor, e, em caso de artrite grave, podem também ser utilizadas doses baixas de corticosteróides $^{(24)}$. Como alternativa, a atividade da doença pode ser controlada com uma combinação de drogas anti-reumáticas modificadoras de doença (DMARDs), tais como metotrexato e hidroxicloroquina. Algumas citocinas, a exemplo do TNF-A e IL-1 são descritas como participantes na proliferação de células sinoviais na $\mathrm{APAH}$, assim como ocorre na $\mathrm{AR}^{(24,25)}$. Portanto, agentes anticitocinas ou antagonistas de receptor de citocinas (IL-1 ou de TNF-A) podem ser eficazes na APAH.

Tem sido demonstrado que os sinoviócitos e células mononucleares articulares expressam antígenos Fas/Fas $\mathrm{L}$, que induzem apoptose ${ }^{(26)}$. Este achado levou à possibilidade do uso do anticorpo anti-Fas como uma nova perspectiva terapêutica. Fujisawa et $a^{(27)}$ observaram que a administração intra-articular de anticorpo anti-Fas em camundongos transgênicos para o gene tax do HTLV-1, inibiu a proliferação de sinoviócitos e células mononucleares, melhorando a artrite.

Outros estudos sugerem que a terapia gênica baseada na inibição da expressão do RNA mensageiro da proteína tax do HTLV-1 pela ribozima pode inibir o crescimento das células sinoviais infectadas pelo HTLV-1, tornando-se uma perspectiva futura para o tratamento de doenças reumáticas associadas ao vírus ${ }^{(28)}$.

\section{ARTRITE REUMATÓIDE}

A AR clássica é uma doença crônica, incapacitante, acometendo aproximadamente $1 \%$ da população mundial ${ }^{(29)}$. A etiopatogênese desta doença ainda não está elucidada. Sabe-se que é de natureza auto-imune e caracterizada por inflamação crônica do tecido sinovial, a qual leva à destruição $\operatorname{articular}^{(30)}$. Estudos sugerem o envolvimento do HTLV-1 como agente desencadeador e perpetuador das doenças inflamatórias articulares crônicas ${ }^{(21)}$. Nishioka et al ${ }^{(31)}$ foram os primeiros autores a propor uma associação entre HTLV-1 e $\mathrm{AR}$, baseando-se na observação de que pacientes portadores de HTLV-1, com e sem mielopatia, apresentavam artropatia crônica inflamatória.

Em estudo realizado em Nagasaki, área endêmica em HTLV-1, a soroprevalência deste vírus em mulheres portadoras de AR foi de 20,4\%, valor significantemente maior do que nas mulheres infectadas doadoras de sangue ou mulheres não-infectadas com $\mathrm{AR}$ doadoras de sangue $(4,2 \%)^{(21)}$. Em outro estudo realizado em Tsushima, uma ilha localizada ao noroeste da ilha Kujushu, no Japão, onde 26,1\% dos habitantes encontram-se infectados pelo HTLV-1, a incidência de sintomas de poliartrite foi de $37 \%^{(5)}$. Estes autores demonstraram também que a prevalência de AR entre os pacientes soropositivos para o HTLV-1 foi de $0,56 \%$, significativamente maior que na população soronegativa $(0,31 \%)$. O mecanismo proposto para este aumento da freqüência de AR em indivíduos infectados pelo HTLV-1 foi de que os linfócitos infectados produziriam maior quantidade de citocinas, tais como IL-1 B, IL-6 e TNF-A que estimularia a proliferação das células sinoviais. A detecção de proteínas do HTLV-1, não somente em células mononucleares, mas também em células do estroma sinovial ${ }^{(32)}$, pode significar uma evidência de que esse vírus tem tropismo para a sinóvia. Iwakura et $\mathrm{a}^{(33)}$ observaram que camundongos transgênicos expressando o genoma do HTLV-1 desenvolvem artrite crônica com proliferação sinovial, formação de pannus, derrame e destruição da cartilagem com erosão articular semelhante à AR de humanos.

\section{SÍNDROME DE SJÖGREN}

A síndrome de Sjögren (SS) primária é definida como distúrbio auto-imune sistêmico, que se manifesta principalmente por xerostomia (boca seca) e xeroftalmia (ceratoconjuntivite seca) devido à infiltração linfocitária das glândulas salivares e lacrimais ${ }^{(34,35)}$.

Vernant et $\mathrm{al}^{(36)}$ foram os primeiros a propor uma associação entre HTLV-1 e SS. O referido autor tomou como base o fato de que pacientes infectados pelo HTLV-1 associado à mielopatia/paraparesia espástica tropical (MAH/PET), comumente desenvolviam sintomas semelhantes aos que ocorrem na SS. Eguchi et $a^{\left({ }^{37)}\right.}$ e Terada et $a^{(38)}$, posteriormente, reportaram alta soroprevalência de HTLV-1 entre pacientes com SS em área endêmica para HTLV-1 (a razão de chances de infecção pelo HTLV-1 ajustada pela idade entre pacientes com SS doadores de sangue foi 3:1).

Os níveis de imunoglobulinas e de auto-anticorpos estão elevados na SS primária. Em 75\% dos casos, encontra-se um FR positivo. Anticorpos antinucleares (ANA) também estão presentes na maioria dos pacientes, assim como os auto-anticorpos nucleares anti-Ro (SS-A) e anti-La (SS-B). Os auto-anticorpos antiducto salivar também podem ser demonstrados, principalmente, na SS secundária ${ }^{(35)}$. A despeito da presença de auto-anticorpos, tem sido aventado que a patogênese da SS se relaciona principalmente constituído por células T/auxiliadoras. Nakamura et $\mathrm{a}^{\left({ }^{(39)}\right.}$ recentemente observaram uma maior densidade de infiltração de células mononucleares em glândulas salivares de pacientes com SS associado à infecção pelo HTLV-1, quando comparado com aqueles não-infectados, porém, o volume de saliva e 
freqüência de anticorpos antinucleares, anti-SSA e anti-SSB não foram diferentes nos dois grupos estudados.

\section{LÚPUS ERITEMATOSO SISTÊMICO}

Lúpus eritematoso sistêmico (LES) é uma doença multifatorial, caracterizada por hiper-reatividade de células B com hipergamaglobulinemia e formação de auto-anticorpos patogênicos. Os pacientes podem também apresentar alterações na proporção de células $\mathrm{T}$ auxiliadoras/supressoras, assim como disfunção de células $\mathrm{T}$, incluindo diminuição numérica, aumento de apresentação antigênica e alteração de respostas para mitógenos, além do aumento na produção de algumas citocinas ${ }^{(40)}$. Embora a etiologia do LES permaneça incerta, além dos fatores genéticos, há evidências de que fatores ambientais a exemplo de infecções retrovirais estejam também envolvidos ${ }^{(41)}$.

Vários pesquisadores têm examinado o possível envolvimento de HTLV-1 no desenvolvimento do LES e os resultados são conflitantes. A descoberta de partículas semelhantes ao HTLV-1 no lúpus murino sugere uma associação entre viroses e LES. Olsen et $a^{42)}$ observaram expressão de antígenos do HTLV-1 em células mononucleares de sangue periférico de indivíduos com LES infectados pelo HTLV-1 após três dias ou mais de cultura in vitro. Foi também observada atividade de transcriptase reversa em sobrenadantes de culturas de células destes indivíduos. Estas são evidências de replicação viral em pacientes com LES e pode explicar a alta soropositividade para HTLV-1 e HTLV-2 nestes pacientes. Ranki et $a^{(43)}$ demonstraram que a ocorrência de anticorpos contra proteínas retrovirais humanas está associada com lesões cutâneas graves e infecções recorrentes no LES, lúpus discóide e doença mista do tecido conjuntivo. Outros trabalhos falharam em determinar associação entre LES e soropositividade para HTLV-1. Murphy et al ${ }^{44)}$ encontraram, em um estudo realizado na Jamaica, área endêmica para o vírus, apenas $6,3 \%$ de soropositividade para HTLV-1 entre pacientes com diagnóstico de LES. Bailer et al ${ }^{45)}$ encontraram sorologia negativa para HTLV-1 em 115 pacientes com

\section{REFERÊNCIAS}

1. Bangham CR, Hall SE, Jeffery KJ, et al: Genetic control and dynamics of the cellular immune response to the human T-cell leukaemia virus HTLV-1. Philos Trans R Soc Lond B Biol Sci 354: 691-700, 1999.

2. Carvalho EM, Bacellar O, Porto AF, Braga S, Galvao-Castro B, Neva F: Cytokine profile and immunomodulation in asymptomatic diagnóstico de doenças auto-imunes do tecido conjuntivo, incluindo LES e AR.

Um mecanismo proposto para a associação entre LES e infecção pelo HTLV-1 é o envolvimento da seqüência endógena relacionada ao HTLV-1 (HRES-1) no desenvolvimento de LES $^{(46)}$. O mimetismo molecular entre HRES-1 e o complexo ribonucleoproteína menor, provocaria a produção de auto-anticorpos, levando a formação de complexos imunes que se depositam nos tecidos, fixação de complemento e inflamação, características patogênicas do $\operatorname{LES}^{(41)}$.

\section{POLIMIOSITE EM INDIVÍDUOS INFECTADOS PELO HTLV-1}

O comprometimento das células teciduais musculares em indivíduos infectados pelo HTLV-1 parece ser conseqüência da resposta imune do hospedeiro às proteínas virais presentes dentro dos macrófagos nos tecidos-alvo ${ }^{(47)}$, constituindo uma evidência da indução de miosite por este retrovírus. Portanto, a polimiosite associada ao HTLV-1 é uma conseqüência do processo imunológico mediado por célula $\mathrm{T}$, dirigido para células infectadas pelo HTLV-1 ${ }^{(48)}$.

\section{CONCLUSÃO}

Existem alguns trabalhos na literatura demonstrando que a infecção pelo HTLV-1 pode levar ao desenvolvimento de doenças auto-imunes, incluindo as doenças reumáticas. No Brasil, apesar de alguns estados constituírem-se em áreas endêmicas importantes para o HTLV-1, existem poucos trabalhos estimando a freqüência das principais doenças reumáticas em indivíduos infectados pelo vírus. A presença da AR e da síndrome de Sjogren em pacientes HTLV-1 positivos com e sem mielopatia/paraparesia espástica tropical, permite supor que essas doenças possam ter um fator etiopatogênico comum num número significativo de casos.

Declaramos a inexistência de conflitos de interesse. human T-lymphotropic virus type 1-infected blood donors. J Acquir Immune Defic Syndr 27: 1-6, 2001.

3. Fugo K, Yoshiki T: Retroviruses and autoimmune diseases. Nipon Rinsho 57: 310-5, 1999.

4. Taniguchi A, Takenaka Y, Noda Y, et al: Adult T cell leukemia presenting with proliferative synovitis. Arthritis Rheum 31: 10767,1988 . 
5. Motokawa S, Hasunuma T, Tajima K, et al: High prevalence of arthropathy in HTLV-1 carriers on a Japanese island. Ann Rheum Dis 55: 193-5, 1996.

6. Brand A, Griffiths DJ, Herve C, Mallon E, Venables PJ: Human retrovirus - 5 in rheumatic disease. J Autoimmun 13: 149-54, 1999.

7. Dourado I, Galvão-Castro B: Human T cell Lymphotropic vírus in Brazil: currents situations in the area with the highest prevalence. AIDS Res Human Retroviruses 17 (supll 9): 017, 2001.

8. Lehky TJ, Cowan CE, Lampson LA, Jacobson S: Induction of HLA class I and class II expression in human T-lymphotropic virus type I-infected neuroblastoma cells. Virol 68: 1854-63, 1994.

9. Aono H, Fujisawa K, Hasunuma T, Marriott SJ, Nishioka K: Extracellular human $\mathrm{T}$ cell leukemia virus type I tax protein stimulates the proliferation of human synovial cells. Arthritis Rheum 41: 1995-2003, 1998.

10. Iwakura Y, Saijo S, Kioka Y, et al: Autoimmunity induction by human $\mathrm{T}$ cell leukemia virus type 1 in transgenic mice that develop chronic inflamatory arthropathy resembling rheumatoid arthritis in humans. J Immunol 55: 1588-98, 1995

11. Mori N, Shirakawa F, Abe M, et al: Human T cell leukemia virus type I tax transactivates the interleukin- 6 gene in human rheumatoid synovial cells. J Rheumatol 22: 2049-54, 1995.

12. Saijo S, Asano M, Horai R, Yamamoto H, Iwakura Y: Supression of autoimmune arthritis in interleukin- 1 deficient mice in which T cell activation is impaired due to low levels of CD40 ligand and OX40 expression on T cells. Arthritis Rheum 46: 533-44, 2002 .

13. Kishi S, Saijyo S, Arai M: Resistance to Fas-mediated apoptosis of peripheral T cells in human T lymphocyte virus type 1 (HTLV-1) transgenic mice with autoimmune arthropathy. J Exp Med 186: 57-64, 1997

14. Kotani M, Tagawa Y, Iwakura Y: Involvement of autoimmunity against type II collagen in the development of arthritis in mice transgenic for the Human T Cell Leukemia virus type I tax gene. Eur J Immunol 29: 54-64, 1999

15. Khoa ND, Hasunuma T, KobataT, Kato T, Nishioka K: Expression of murine HOXD9 during embryonic joint patterning and in human $\mathrm{T}$ lymphotropic virus type 1 tax transgenic mice with arthropathy resembling rheumatoid arthritis. Arthritis Rheum 42: 686-96, 1999.

16. Levin MC, Lu SM, Kalume F, et al: Autoimmunity due to molecular mimicry as a cause of neurological disease. Nat Med 8: 509-13, 2002.

17. McCallum RM, Patel DD, Moore JO, Haynes BF: Arthritis syndromes associated with human $\mathrm{T}$ cell lymphotropic virus type 1 infection. Med Clin North Am 81: 261-76, 1997.

18. Harden EA, Moore JO, Haynes BF: Leukemia-associated arthritis: identification of leukemic cells in synovial fluid using monoclonal and polyclonal antibodies. Arthritis Rheum 27: 1306-8, 1984.

19. Sato K, Maruyama I, Maruyama Y, et al: Arthritis in patients infected with human T lymphotropic virus type I. Clinical and immunopathologic features. Arthritis Rheum 34: 714-721, 1991.

20. Nishioka K, Nakajima T, Hasunuma T, Sato K: Rheumatic
Manifestation of Human Leukemia Virus Infection. Rheum Dis Clin North Am 19: 489-503, 1993.

21. Eguchi K, OriguchiT, Takashima H, Iwata K, Katamine S, Nagataki S: High Seroprevalence of Anti-HTLV-1 Antibody in Rheumatoid Arthritis . Arthritis Rheum 39: 463-6, 1996.

22. Nishioka K: HTLV-I arthropathy and Sjögren syndrome. J Acquir Immune Defic Syndr Hum Retrovirol. Review 13 (Suppl 1): $557-62,1996$.

23. Yin W, Hasunuma T, Kobata T, Sumida T, Nishioka K: Synovial hyperplasia in HTLV-1 associated arthropathy is induced by tumor necrosis factor-alpha produced by HTLV-1 infected CD68+ cells. J Rheumatol 27: 874-81, 2002.

24. Hasunuma T, Sumida T, Nishioka K: Human T cell leukaemia virus type I and rheumatoid arthritis. International Review of Immunology 17(5-6): 291-307, 1998.

25. Moss RB, Moll T, El-Kalay M, et al: Th1/Th2 cells in inflammatory disease states: therapeutic implications. Expert Opin Biol The 4: 1887-96, 2004.

26. Kobayashi T, Okamoto K, Kobata T, et al: Apomodulation as a novel therapeutic concept for the regulation of apoptosis in rheumatoid synoviocytes. Curr Opin Rheumatol 11: 188-93, 1999.

27. Fujisawa K, Asahara H, Okamoto K, et al: Therapeutic effect of the anti-Fas antibody on arthritis in HTLV-I tax transgenic mice. J Clin Invest 98: 271-78, 1996.

28. Kitajima I, Hanyu N, Kawahara K, et al: Ribozyme-based gene cleavage approach to chronic artritis associated with human $\mathrm{T}$ cell leukemia virus type I: induction of apoptosis in synoviocytes by ablation of HTLV-1 tax protein. Arthritis Rheum 40: 2118-27, 1997.

29. Iwakura Y: Mouse Models for Rheumatoid Arthritis and their use in drug development. Nippon Yakurigaku Zasshi 120: 303-13, 2002.

30. Firestein GS, Zvaifler NJ: Rheumatoid Arthritis: A disease of disordered immunity. In inflammation: Basic Principles and Clinical Correlates, eds. Raven Press, New York, 959, 1992.

31. Nishioka K, Kitajima I, Yamamoto K, et al: Novel mechanism of synovial proliferation caused by human T Leukemia / lymphotropic virus type 1 (abstract). Arthritis Rheum 34 (suppl 9): S33, 1991 .

32. Nishioka K, Sumida T, Hasunuma T: Human T lymphotropic virus type $\mathrm{I}$ in arthropathy and autoimmune disorders. Arthritis Rheum 39: 1410-8, 1996.

33. Iwakura Y, Tosu M, Yoshida E: Induction of inflammatory arthropathy resembling rheumatoid arthritis in mice transgenic for HTLV-1. Science 253: 1026-28, 1991.

34. Lê Toux G: Pathologie Revue d'odonto - Stomatologie.Tome 21: 285-96, 1992

35. Fox RI, Tornwall J, Maruyama T, Stern M: Evolving concepts of diagnosis, pathogenesis, and therapy of Sjogren's syndrome. Curr Opin Rheumatol 10: 446-56, 1998.

36. Vernant JC, Buisson G, Magdeleine J, et al: T-lymphocyte alveolitis, tropical spastic paresis, and Sjogren syndrome. Lancet 1: 177(Letter), 1988.

37. Eguchi K, Matsuoka N, Ida H, et al: Primary Sjögren's syndrome with antibodies to HTLV-1: clinical and laboratory features. Ann Rheum Dis 51: 769-76, 1992.

38. Terada K, Katamine S, Eguchi K, et al: Prevalence of serum and 
salivary antibodies to HTLV-1 in Sjögren 's syndrome. Lancet 344: 1116-9, 1994.

39. Nakamura H, Eguchi K, Nakamura T, et al: High prevalence of Sjögren's syndrome in patients with HTLV-1 associated myelopathy. Ann Rheum Dis 56: 167-72, 1997.

40. Lipka K, Tebbe B, Finckh U, Rolfs A: Absence of Human T - Lymphotrophic virus type I in patients with systemic lupus erythematosus. Clin Exp Dermatol 21: 38-42, 1996.

41. Adelman MK, Marchalonis JJ: Endogenous Retroviruses in Systemic Lupus Erythematosus: candidate lupus viruses. Clin Immunol 102: 107-16, 2002.

42. Olsen RG, Tarr MJ, Mathes LE, et al: Serological and virological evidence of human $\mathrm{T}$ - lymphotropic virus in systemic lupus erythematosus. Med Microbiol Immunol (Berl) 176: 53-64, 1987.

43. Ranki A, Kurki P, Riepponen S, Stephansson E: Antibodies to retroviral proteins in autoimmune connective tissue disease. Relation to climical manifestations and ribonucleoprotein autoantibodies. Arthritis Rheum 35: 1483-91, 1992.
44. Murphy Jr EL, De Ceulaer K, Williams W, et al: Lack of relation between human T-lymphotropic virus type I infection and systemic lupus erythematosus in Jamaica, West Indies. J Acquir Immune Defic Syndr 1: 18-22, 1988.

45. Bailer RT, Lazo A, Harisdangkul V, et al: Lack of evidence for human $\mathrm{T}$ cell lymphotropic virus type 1 or 2 infection in patients with systemic lupus erythematosus or rheumatoid arthritis. J Rheumatol 21: 2217-24, 1994.

46. Magistrelli C, Samoilova E, Agarwall RK, et al: Polymorphic genotypes of the HRES-1 human endogenous retrovirus locus correlate with systemic lupus erythematosus and autoreactivity. Immunogenetics 49: 829-34, 1999.

47. Itescu S: Rheumatic aspects of acquired immunodeficiency syndrome. Curr Opin Rheumatol 8: 346-53, 1996.

48. Higushi $\mathrm{M}$, Nagasawa $\mathrm{K}$, Horiuchi $\mathrm{T}$, et al: Membrane tumor necrosis factor-alpha (TNF-alpha) expressed on HTLV - I infected $\mathrm{T}$ cells mediates a costmulatory signal for B cell activationcharacterization of membrane TNF-alpha. Clin Immunol Immunopathol 82: 133-40, 1997. 